
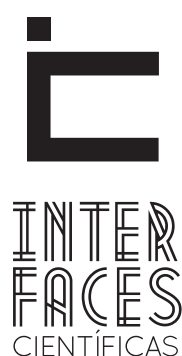

HUMANASE SOCIAIS

ISSN IMPRESSO 2316-3348

E-ISSN 2316-3801

DOI - 10.17564/2316-3801.2017v6n1p119-126

\title{
A PSICOLOGIA NO CONTEXTO DAS POLÍTICAS PÚBLICAS DE ASSISTÊNCIA SOCIAL
}

\author{
PSYCHOLOGY IN THE CONTEXT OF PUBLIC POLLCY
}

PSICOLOGÍA EN EL CONTEXTO DE LA POLLITICA PÚBLICA

\section{RESUMO}

A pesquisa teve por objetivo analisar o papel do psicólogo dentro do Centro de Referência em Assistência Social (CRAS), com enfoque em aspectos relevantes à sua atuação nas políticas públicas. 0 presente trabalho se torna importante porque busca-se mostrar em que contexto se encontra inserido o psicólogo, que atua na comunidade e para a comunidade, que assume assim, um compromisso: conhecer sua realidade e compreender os modos de subjetivação contemporâneos. Desta forma, para a obtenção do objetivo previsto, utilizou-se como recurso metodológico, a pesquisa de revisão sistemática, realizada a partir da leitura de aparatos já publicados na lite- ratura e artigos científicos divulgados no meio impresso e eletrônico. A partir disso, percebeu-se que as principais atividades do Psicólogo são: atendimento psicossocial e acompanhamento as famílias; inserção das famílias em benefícios, programas de geração de renda; elaboração de laudo, relatório e parecer psicossocial; atividade socioeducativa e cursos profissionalizantes.

\section{PALAVRAS-CHAVE}

Psicólogo. Centro de Referência em Assistência Social. Subjetivação. 


\section{ABSTRACT}

The research aimed to analyze the role of psychologists in the Reference Center for Social Assistance CRAS ), focusing on aspects relevant to its activities in public policy. This study becomes important because we seek to show in what context is inserted the psychologist who works in the community and for the community, and thus assumes a commitment: to know their reality and understand the ways of contemporary subjectivity. Thus, for the full achievement of expected goals, it was used as a methodological resource, the systematic review of research conducted from the apparatus read previously published in the literature and scientific ar- ticles published in the printed and electronic media. From this, it was noticed that the main activities of the Psychologist are: psychosocial care and monitoring of families; insertion of families into benefits, in come generation programs; preparation of report, reportand psychosocial opinion; socio-educational activity and vocational courses.

\section{KEYWORDS}

Psychologist. Reference Center for Social Assistance. Subjectivity .

\section{RESUMEN}

La investigación tuvo como objetivo analizar el papel de los psicólogos en el Centro de Referencia de Asistencia Social (CRAS), centrándose en aspectos relevantes para sus actividades en la política pública. Este estudio se convierte en importante, ya que tratamos de mostrar en qué contexto se inserta el psicólogo que trabaja en la comunidad y para la comunidad, y por lo tanto asume un compromiso: para conocer su realidad y comprender las formas de subjetividad contemporánea. Por lo tanto, para la plena consecución de los objetivos previstos, se utiliza como recurso metodológico, la revisión sistemática de la investigación llevada a cabo desde publicaciones anteriores en la bibliografía y artículos científicos publicados en los medios de comunicación impresos y electrónicos. A partir de eso, se percibió que las principales actividades del Psicólogo son: atención psicosocial y acompañamiento a las familias; inserción de las familias en beneficios, programas de generación de ingresos; elaboración de laudo, informe y opinión psicosocial; la actividad socioeducativa y los cursos de formación profesional.

\section{PALABRAS CLAVE}

Psicólogo. Centro de Referencia de Asistencia Social. Subjetividad. 


\section{INTRODUÇÃO}

De acordo com as diretrizes estabelecidas pela Constituição de 1988, pode-se destacar a promulgação em 1993 por meio da Lei Orgânica da Assistência Social - LOAS (Lei no 8.742/93), que interrompe: "A visão assistencialista, rejeita a tutela da população usuária dos serviços, abrindo possibilidades de desenvolvimento da autonomia e do protagonismo dos sujeitos, por meio das oportunidades de acesso a benefícios e serviços" (ARREGUI, 2007, p. 120).

A Política Pública da Assistência Social visa garantir a todos os que dela necessitarem, sem contribuição prévia, a proteção social. A concepção da assistência social como política e como direito à proteção e à seguridade social por meio da Constituição de 1988 trouxe uma nova abordagem a essa política, objetivando desvinculá-la dos tradicionais modelos assistencialistas e compreendendo-a como garantidora dos direitos da cidadania (BRASIL, 1988). Sendo um direito do cidadão, pretende prover os mínimos direitos sociais para garantir as necessidades básicas, a segurança de sobrevivência (rendimento e autonomia), a segurança da acolhida (alimentação, vestuário e abrigo) e o convívio familiar.

Suas diretrizes baseiam-se na descentralização das ações, sendo de responsabilidade da esfera federal a coordenação e as normas gerais, dos Estados e Municípios a coordenação e a execução dos programas e projetos, garantindo-se o comando das ações em cada esfera de governo, respeitando-se as diferenças e as características territoriais locais (LOPES, 2007).

O Sistema Único da Assistência Social (SUAS), implantado em 2005, no País, define e organiza toda a referida política, reordenando a gestão, visando a ações descentralizadas e participativas em todo o Brasil (BRASIL, 2006). O SUAS estabelece ainda duas formas de proteção social, que se ocupam das vulnerabilidades e riscos que os cidadãos enfrentam em sua trajetória de vida. A proteção social básica e a proteção social especial compõem essas duas formas, sendo a primeira responsável por prevenir situações de risco e a segunda intervém em casos em que há situações de risco com ou sem rompimento dos vínculos familiares (ARREGUI, 2007).

A partir disso, o autor Lopes (2007) destaca que o trabalho do psicólogo está atrelado a interpretação e a sinalização ao indivíduo, de modo que se identificam os significantes que ele poderá se apoderar como seus e os que ele reproduzirá sem a tomada de consciência.

Dessa forma, o presente artigo tem como objetivo analisar o papel do psicólogo dentro do Centro de Referência em Assistência Social (CRAS), com enfoque em aspectos relevantes à sua atuação nas políticas públicas.

O motivo norteador que impulsiona pesquisar essa temática é o fato de perceber-se, a partir de leituras que muitas vezes a sociedade não sabe qual o papel do psicólogo dentro das políticas públicas, confundindo com o trabalho do Assistente Social.

0 tema tratado neste artigo é relevante porque a delimitação da função do psicólogo no assistencialismo social é algo muito debatido nos últimos anos. Por estar na era da revolução digital, faz-se necessário que essa delimitação se perpetue por todos os meios de informações.

Dessa forma, para alcançar o objetivo proposto, utilizou-se como recurso metodológico, a pesquisa de revisão sistemática, realizada a partir da leitura de aparatos já publicados na literatura e artigos científicos divulgados no meio impresso e eletrônico. Vários foram os autores utilizados para a construção teórica do trabalho, dentre eles estão: Arregui (2007), Botarreli (2008), Carvalho (2011), Costa (2015) e tantos outros.

\section{DESENVOLVIMENTO}

\subsection{CONSTITUIÇÃO FEDERAL DE 1988, LEI ORGÂNICA DA ASSIS- TÊNCIA SOCIAL-LOAS/1993 E A POLÍTICA NACIONAL DE ASSIS- TÊNCIA SOCIAL - PNAS/2004}

Para compreender a forma de organização da Política de Assistência Social no Brasil, seus limites e contradições, é importante analisar sua trajetória até chegar a Constituição Federal de 1988, para entender como se deu esse processo de transformações que 
culminou com o reconhecimento desta como uma política pública, tornando-se um direito que, junto com a saúde e previdência social, formam o tripé da seguridade social (FERREIRA, 2013).

No Brasil, até a década de 1930, não havia uma intervenção do Estado na área social, toda prática assistencial que existia até esse momento era desenvolvida pela Igreja Católica e organizações de caridade. As práticas assistenciais tinham um caráter disciplinador e não havia uma compreensão da pobreza como expressão da questão social, ela era vista como uma disfunção individual e tratada como caso de polícia por meio do aparato repressor do Estado. Somente a partir da Revolução de 1930, quando Getúlio Vargas chega à presidência do país, que o Estado começa a assumir novas funções, deixando de ser apenas coercitivo (MESTRINER, 2008).

Mas, somente a partir da Constituição Federal de 1988, regulamentada pela Lei $n^{0} 8.742$, de 7 de dezembro de 1993, intitulada Lei Orgânica da Assistência Social, novos conceitos e modelos de assistência social vigoraram no Brasil, sendo esta colocada como direito de cidadania, com vistas a garantir o atendimento às necessidades básicas dos segmentos populacionais vulnerabilizados pela pobreza e pela exclusão social (PIRES, 2013).

A Constituição Federal de 1988 foi aprovada no dia 5 de outubro e traz a Política de Assistência Social inscrita nos artigos 203 e 204: Art.203 A Assistência Social será prestada a quem dela necessitar, independentemente de contribuição à seguridade social e tem por objetivos:

I- a proteção à família, à maternidade, à infância, à adolescência e à velhice;

II- o amparo às crianças e adolescentes carentes;

III- a promoção da integração ao mercado de trabalho;

IV- a habilitação e a reabilitação das pessoas portadoras de deficiência e a promoção de sua integração à vida comunitária;

V- a garantia de um salário mínimo de benefício mensal à pessoa portadora de deficiência e ao idoso que comprovem não possuir meios de prover à própria manutenção ou de tê-la provida por sua família, conforme dispuser a lei.

A partir da Constituição Federal de 1988, foi regulamentada a Lei no 8.742, de 7 de dezembro de 1993, intitulada Lei Orgânica da Assistência Social, na qual, novos conceitos e modelos de assistência social passaram a vigorar no Brasil, sendo esta colocada como direito de cidadania, com vistas a garantir o atendimento às necessidades básicas dos segmentos populacionais vulnerabilizados pela pobreza e pela exclusão social (PIRES, 2013).

Posteriormente a Lei Orgânica da Assistência Social, tem-se a elaboração da Política Nacional de Assistência Social conquistada após a IV Conferência Nacional de Assistência Social realizada em dezembro de 2004, tendo como significativo avanço a efetivação do sistema descentralizado e participativo a Política de Assistência Social. A partir de então a Assistência Social está definitivamente inserida no campo das políticas públicas brasileiras (MAGALHÃES; SILVA; OLIVEIRA, 2012).

A Política Nacional de Assistência Social tem seus princípios, diretrizes, objetivos e mecanismos criados em consonância com a LOAS, entre os quais são princípios:

I - Supremacia do atendimento às necessidades sociais sobre as exigências de rentabilidade econômica;

II - Universalização dos direitos sociais, a fim de tornar o destinatário da ação assistencial alcançável pelas demais políticas públicas;

III - Respeito à dignidade do cidadão, à sua autonomia e ao seu direito a benefícios e serviços de qualidade, bem como à convivência familiar e comunitária, vedando-se qualquer comprovação vexatória de necessidade;

IV - Igualdade de direitos no acesso ao atendimento, sem discriminação de qualquer natureza, garantindo-se equivalência às populações urbanas e rurais;

V - Divulgação ampla dos benefícios, serviços, 
programas e projetos assistenciais, bem como dos recursos oferecidos pelo Poder Público e dos critérios para sua concessão. (BRASIL, 2005, p. 26).

A Política Nacional de Assistência Social tem um caráter inovador, pois avança no sentido de se consumar como política pública que reconhece a questão social como uma situação coletiva da sociedade capitalista e busca superar as práticas focalizadas e assistencialistas (LOPES, 2007).

\subsection{SISTEMA ÚNICO DE ASSISTÊNCIA SOCIAL - SUAS}

Um novo avanço que se apresenta, a partir de 2005, é a implantação do Sistema Único da Assistência Social (SUAS). Sua proposta de implantação vem desde a promulgação da LOAS e da I Conferência Nacional de Assistência Social (LOPES, 2007).

A implantação do SUAS como sistema único supõe unir para garantir, o que implica em: romper com a múltipla fragmentação programática hoje existente, com a fragmentação das esferas de governo e o paralelismo de gestão; com a fragmentação das ações por categorias ou segmentos sociais sem compromisso com a cobertura universal e a qualidade dos resultados (SPOSATI, 2007).

Verifica-se o cuidado da Política Nacional de Assistência Social em respeitar os cidadãos e as famílias reconhecendo-os como sujeitos de sua própria história, dotados de experiências e valores e capazes de construir mudanças. A proteção social de assistência social segundo a NOB/SUAS (2005, p. 16):

[...] consiste no conjunto de ações, cuidados, atenções, benefícios e auxílios ofertados pelo SUAS para redução e prevenção do impacto das vicissitudes sociais e naturais ao ciclo da vida, à dignidade humana e à família como núcleo básico de sustentação afetiva, biológica e relacional.

Portanto, a proteção tem por princípios a matricialidade sociofamiliar, a territorialização, a proteção pró-ativa, a integração à seguridade social e a integração às políticas sociais e econômicas, tem ainda por garantia a segurança de acolhida, a segurança social de renda, a segurança do convívio ou vivência familiar, comunitária e social, a segurança do desenvolvimento da autonomia individual, familiar e social e a segurança de sobrevivência a riscos circunstanciais (NOBREGA et al., 2009).

\subsection{CENTRO DE REFERÊNCIA EM ASSISTÊNCIA SOCIAL [CRAS]}

Os CRAS são, prioritariamente, os responsáveis pela execução dos serviços, programas, projetos e benefícios de proteção social básica, além da organização e coordenação da rede de serviços socioassistenciais locais (COSTA; AGUIAR, 2015).

\begin{abstract}
O Centro de Referência de Assistência Social (CRAS) é uma unidade pública estatal descentralizada da política de assistência social, responsável pela organização e oferta de serviços da proteção social básica do Sistema Único de Assistência Social (SUAS) nas áreas de vulnerabilidade e risco social dos municípios e DF. Dada sua capilaridade nos territórios, se caracteriza como a principal porta de entrada do SUAS, ou seja, é uma unidade que possibilita o acesso de um grande número de famílias à rede de proteção social de assistência social. (BRASIL, 2006, p. 9).
\end{abstract}

Nestes centros, as ações direcionam-se às pessoas e/ou famílias em situação de vulnerabilidade social, seja em razão do rompimento e/ou fragilização dos vínculos afetivos familiares ou comunitários, desemprego, pobreza, entre outros (LOPES, 2007). O CRAS possui duas funções específicas: gestão da proteção básica no seu território e de oferta do Programa de Atenção Integral à Família (PAIF). Cabe ressaltar que estes serviços só podem ser oferecidos pelo Centro de Referência de Assistência Social (MAGALHÃES; SILVA; OLIVEIRA, 2012).

Esta unidade pública possibilita o atendimento descentralizado, dentro das próprias comunidades e/ ou bairros, com ações propostas a partir da realidade apresentada e real necessidade da população usuária. Sua instalação deve se dar em áreas de maior concentração de vulnerabilidade social, para isso, é fundamenta a identificação destas por meio de diagnóstico previamente realizado (CARVALHO, 2011). 
A equipe do CRAS, composta por psicólogos, assistentes sociais, auxiliar administrativo, dentre outros profissionais, deve combater a exclusão e favorecer "[...] a inclusão e a equidade dos usuários e grupos específicos, ampliando o acesso aos bens e serviços socioassistenciais básicos e especiais, em áreas urbana e rural [...]" (BRASIL, 2006, p.33).

\subsection{PSICÓLOGO NO CONTEXTO DO CRAS}

Segundo o Centro de Referência Técnica em Psicologia e Políticas Públicas (CREPOP), criado em 2006 com o objetivo de proporcionar um novo olhar e oferecer qualidade ao trabalho do psicólogo nas políticas públicas, a atuação do psicólogo como trabalhador da Assistência Social tem como finalidade básica o fortalecimento dos usuários como sujeitos de direitos.

A implantação do CRAS nas comunidades que apresentam maior vulnerabilidade social implica em maiores possibilidades para essas famílias atendidas, assim como também se torna uma ferramenta importante para que o psicólogo trabalhe em consonância com as necessidades sociais. 0 trabalho desenvolvido pelo psicólogo no CRAS é fundamental, a título exemplificativo tem-se a promoção de uma atuação interdisciplinar com toda a equipe de profissionais envolvidos neste núcleo, dentre outros papéis demasiadamente importantes. Contudo, um dos principais focos do psicólogo no CRAS é o atendimento psicossocial, trabalho realizado junto ao assistente social e que abandona o caráter clínico, tendo um caráter familiar/sistêmico, com uma visão especialmente direcionada a grupalidade (NOBREGA et al., 2009).

O CREPOP (2007) aponta a importância de conhecer o sujeito, assim como fazê-lo se reconhecer diante da sua realidade. Atribui aos profissionais que atuam neste âmbito o auxílio a esses sujeitos em enxergar e valorizar a capacidade que possuem de se transformar socialmente.

Segundo Brasil (2006), tanto os psicólogos quanto os outros profissionais de nível superior, devem ter conhecimento da legislação que diz respeito à política nacional de assistência social, dominar sobre os direitos sociais, ter experiência de trabalhos em grupos e atividades coletivas, conhecerem a realidade do território, ter bom relacionamento interpessoal e saber realizar uma escuta qualificada às famílias.

No modelo de psicologia que se propõe nesta atual política, o psicólogo contribui para a formação da cidadania num caráter de elaboração de uma consciência junto ao sujeito e a comunidade, busca essa conscientização acerca das possibilidades de ser. 0 labor do psicólogo fundamenta-se no que é estruturante na vida da comunidade (NOBREGA et al., 2009).

Segundo Lopes (2007, p. 32) "o psicólogo pode interpretar e sinalizar ao indivíduo quais significantes ele pode se apoderar como seus e quais são os que ele reproduz sem tomada de consciência”, assim a comunidade tomará posse desses significantes que a caracterizam, desenvolvendo as atividades no CRAS, produzindo conhecimento nos grupos. A partir desse processo surge a autonomia de se apoderar do que lhe convém, ocorrendo assim a tomada de consciência de um conhecimento produzido por ela, não mais reproduzido ou imposto pela lógica do sistema. É então que emerge a autonomia que a Política Nacional de Assistência Social (PNAS) almeja.

Nesta lógica, Martin-Baró (1996, p. 15) acrescenta:

\begin{abstract}
O saber mais importante do ponto de vista psicológico não é o conhecimento explícito e formalizado, mas esse saber inserido na práxis cotidiana, na maioria das vezes implícito, estruturalmente inconsciente, e ideologicamente naturalizado, enquanto adequado ou não a realidade objetiva, enquanto humaniza ou não as pessoas, e enquanto permite ou impede os grupos e povos de manter o controle de sua própria existência.
\end{abstract}

No tocante a operacionalização do trabalho do psicólogo no CRAS, Botarelli (2008), que recolheu relatos destes profissionais inseridos no campo da proteção social básica, aponta a importância dos grupos socioeducativos para promover encontros que tenham como finalidade facilitar a emancipação, a troca de afetos e o questionamento de influências ideológicas e opressoras que contribuem para a perpetuação do ciclo de desigualdade social, pobreza e alienação das pessoas. 
Neste sentido, o grupo reflexivo é utilizado como um instrumento privilegiado para a análise dos depoimentos e das vivências pessoais dos indivíduos participantes cujas falas são problematizadas para mostrar que uma situação não é tecida apenas por componentes individuais, mas também são geradas pelo contexto social. Além disso, busca estimular uma valorização da rede social, possibilitando reflexões sobre posturas e ações adotadas na vida cotidiana que interferem nos laços sociais tanto no âmbito familiar como em comunidade (BOTARELLI, 2008).

\section{CONCLUSÃO}

Os serviços prestados no CRAS visam a promoção da assistência e a prevenção de problemas assistenciais, trabalhando de forma interdisciplinar para meIhor servir a população dos municípios brasileiros, para se conseguir alcançar um nível máximo de excelência no atendimento, possibilitando aos seus usuários um acesso amplo a todos os Serviços de Assistência Social que estes necessitam.

O Psicólogo revela-se como um instrumento de promoção de direitos e cidadania, de suas ações que são desenvolvidas por meio do trabaIho social com famílias, apreendendo as origens, os significados atribuídos e as possibilidades de enfrentamento das situações de vulnerabilidades vivenciadas, contribuindo para sua proteção de forma integral na perspectiva de que os projetos produzam um novo significado de vida. Além disso, torna-se necessário tentar reverter a realidade de exclusão social vivenciada por estas pessoas em oportunidades de mudança e melhoria na qualidade de vida e esperança de um futuro com dignidade e igualdade de direitos.

O CRAS tem como objetivos: Prevenir situação de risco social (descriminação, desemprego, trabalho infantil, violência). Suas principais atividades são: Atendimento psicossocial e acompanhamento às famílias; Inserção das famílias em benefícios, programas de geração de renda; Elaboração de laudo, relatório e parecer psicossocial; Atividade socioeducativa e cursos profissionalizantes.

0 presente artigo foi proposto para promover a ampliação dos conhecimentos sobre a atuação do psicólogo no CRAS, identificando os principais desafios enfrentados pelos psicólogos nesses Centros, procurando conhecer sua realidade, suas necessidades, melhorando a qualidade de vida das pessoas, reassumindo o pacto com os direitos humanos e utilizando de sua autonomia para defender, criar e fiscalizar as políticas públicas.

\section{REFERÊNCIAS}

ARREGUI, Carola C. Assistência social: controle e política pública. São Paulo: Instituto Polis, 2007.

\section{BOTARELLI, Adalberto. 0 psicólogo nas políticas}

de proteção social: uma análise dos sentidos e das práxis. 2008. 133f. Tese (Doutorado em Psicologia Social) - Pontifícia Universidade Católica de São Paulo, São Paulo, 2008.

\section{BRASIL. Constituição. Constituição da República}

Federativa do Brasil. Brasília-DF: Senado Federal; Centro Gráfico, 1988. 292p.

BRASIL. Ministério do Desenvolvimento Social e Combate à Fome. Norma Operacional Básica: NOB/ SUAS. Brasília, Jul. 2005.

BRASIL. Ministério do Desenvolvimento Social e Combate à Fome. Política Nacional de Assistência Social. Brasília, 2006.

\section{CARVALHO, Maria do Carmo Brant. A família} contemporânea em debate. 2.ed. São Paulo: EDUC; Cortez, 2011.

CREPOP - Centro de Referência técnica em psicologia e políticas públicas. Referência técnica para atuação do (a) psicólogo (a) no CRAS / SUAS. Brasília: Conselho Federal de Psicologia, 2007. 
COSTA, Francilene Soares de Medeiros; AGUIAR, Nuara de Sousa. Centros de Referência da

Assistência Social - CRAS: materializações e contradições da Política Nacional de Assistência Social. São Paulo: UNESP, 2015. 19p. Disponível em: <http://www.cchla.ufrn.br/cnpp/pgs/anais/Arquivos GTS - recebidos em PDF/Centros de Referência da Assistência Social - CRAS materializações e contradições da Política Nacional de Assistência Social.pdf>. Acesso em: 28 maio 2016.

FERREIRA, Andressa do Nascimento. A atuação profissional dos assistentes sociais nos centros de referência de assistência social (CRAS) do Município de São Pedro da Aldeia - RJ. 2013. 77f. TCC (Graduação) - Curso de Serviço Social, Universidade Federal Fluminense - Pólo Universitário de Rio das Ostras, Rio das Ostras, 2013. Disponível em: <http://www.puro.uff.br/tcc/2012-2/Andressa do Nascimento Ferreira.pdf>. Acesso em: 28 maio 2016.

LOPES, José Goes. O psicólogo no CRAS. Revista Psicologia e práticas, UNB, Brasília, v.3, n.1, p.22-32, abril 2007. Disponível em: <http:// revistapsicologiaepraticas.periodicosunb.edu. br/revista/index.php/article/view/4598/4356>. Acesso: 28 maio 2016.

MAGALHÃES, Sabrina da Silva; SILVA, Vanessa Oliveira; OLIVEIRA, Juliene Aglio de. O desafio do fortalecimento dos vínculos familiares e comunitários nos CRAS de Álvares Machado e
Regente Feijó. Seminário Integrado, Presidente Prudente, v.6, n.6, p.1-25, jun. 2012. Disponível em: <http://intertemas.toledoprudente.edu.br/ revista/index.php/Seminariolntegrado/article/ view/4598/4356>. Acesso em: 28 maio 2016.

MARTIN-BARÓ, Ignácio. 0 papel do psicólogo.

Estudos de Psicologia, Natal, n.1, v.2, p.7-27, 1996.

\section{MESTRINER, Maria Luiza. 0 Estado entre a} filantropia e a assistência social. 3.ed. São Paulo: Cortez, 2008.

NÓBREGA, Alex Figueiredo et al. A práxis psicológica nos centros de referência da assistência social. Anais do XV Abrapso, Maceió, v.2, n.1, p.1-8, maio 2009. Disponível em: <http://www.abrapso.org.br/ siteprincipal/images/Anais_XVENABRAPSO/575. a prxis psicolgica nos centros de referncia da assistencia social.pdf>. Acesso em: 28 maio 2016.

\section{PIRES, Izabel Scheidt. Política nacional de} assistência social, suas e legislações pertinentes. São Paulo: Capacitação Suas, 2013. 35 slides, color. Disponível em: <http://www.desenvolvimentosocial. pr.gov.br/arquivos/File/Capacitacao/material_apoio/ mariaizabel_suas.pdf>. Acesso em: 28 maio 2016.

SPOSATI, Aldaíza. A menina Loas: um processo de construção da Assistência Social. 3.ed. São Paulo: Cortez, 2007. 